\title{
Integration of manufacturing system and product design with DMU
}

\author{
Dipl.-Ing. Stefan von Praun \\ Institute for Machine Tools and Industrial Management \\ Technische Universität München \\ Boltzmannstraße 15 / D-85748 Garching / Germany \\ Phone: +49-89-289-15569 Fax: +49-89-289-15555 \\ e-mail:pr@iwb.mw.tu-muenchen.de
}

\begin{abstract}
Digital mock-up (DMU) is a new product and process development strategy that will have a significant impact on the development process of the future. The IT technologies created in DMU research projects are to permit product and process verification in any product development phase, without requiring physical models or prototypes and with only one final verification against the physical model. One of these new technologies is digital mock-up process simulation (DMU-PS).

In future, digital mock-up process simulation is to enable engineers to model and simulate assembly and disassembly processes for series production, after-sales service and recycling within an integrated software system.
\end{abstract}

\section{Keywords}

Digital Mock-up, Manufacturing System Design, Simulation, EDM, PDM, Data Management, Workflow-Management, Business Process

\section{INTRODUCTION}

New technologies are increasingly key to the success of many companies and entire markets. While technology in the past was a derivative of corporate strategy, modern technologies today, such as the Internet, are opening up additional lines of business and ideas which help create new markets. Thus, for instance, information technology (IT) is becoming a driving factor for entire corporate strategies. The performance capabilities of computers and software applications have grown enormously over the past few years and today allow us to take totally new

The original version of this chapter was revised: The copyright line was incorrect. This has been corrected. The Erratum to this chapter is available at DOI: 10.1007/978-0-387-35390-6_58 
approaches in product development. Proven strategies must be subjected to critical scrutiny.

Yet, information technology in this context must never be seen as a stand-alone factor. Only an integrated, holistic approach to strategies, processes, staff and technologies assures survival and allows companies to effectively respond to continually changing markets.

Digital mock-up - a new information technology - is currently a high-profile issue and not only in the automotive and aircraft industries. Only few are aware of the origin and definition of digital mock-up (DMU). In publications and advertising events of software houses, the complexity of a DMU strategy is frequently reduced to collision detection and installation studies. However, the definition of DMU includes a lot more than that.

\subsection{Definition of digital mock-up}

Digital mock-up is a realistic computer simulation of a product and its environment and serves as a platform for product and process development, communication and decision-making from the first product concept all the way to after-sales service and recycling analyses (AIT 1996).

Hence, DMU is a product development strategy which extends far beyond existing approaches. Similar to the way CAD systems today assist design engineers in the modelling, selection, re-use of data and documentation in the product design phase, digital mock-up will enable design engineers, assembly and manufacturing planners as well as management to simultaneously develop product and production. The objective of a digital mock-up strategy is to merge product and production development using simulated models and prototypes. The DMU strategy therefore not only requires new approaches in information technology but also and above all changes in organisation and business processes. The vision behind DMU is this (AIT 1996):

Assessment of product design and serviceability of the product, including the feasibility of production and assembly processes, as part of an integrated software solution with only one final verification against the physical model.

\subsection{Origin of digital mock-up}

The European research initiative AIT (Advanced Information. Technology in Design and Manufacture) was founded in 1993 by the major European automobile manufacturers and aerospace companies and their suppliers. Sponsored by the European Union, AIT has set itself the target to strengthen the competitiveness of the European industry through the development and application of innovative IT technologies.

The aim of the AIT initiative was to close the gap between supply and demand of IT technology. Based on the consensus of the European users in terms of the future requirements for more competitive development and manufacturing processes, the 
necessary research and development projects were set up together with software developers and research institutes.

\subsection{Digital mock-up process simulation}

One of these research projects deals with the subject of process simulation. The complexity of the DMU strategy cannot be reduced to installation simulations and collision detection as part of process simulation (DMU-PS). Process simulation also focuses on such areas as simulation of production, assembly and disassembly in the after-sales service sector as well as of recycling processes. The analysis and simulation of functions, geometry interrelationships, tolerances, ergonomics and logistics are part of a DMU strategy along with process simulation.

Process knowledge is implemented through the use of effective methods and tools as early as during the concept phase and also the subsequent phases of the development process. The specific goal of the DMU-PS research project (DMU-PS 1996) is to develop a technology enabling design engineers, assembly planners and management to develop production processes with digital rather than physical models and prototypes. In deviating from current practice, decisions during the concept, layout and subsequent phases will in future be made on the basis of digital mock-up.

\section{STATE OF THE ART}

The automotive, aircraft and other manufacturing industries are currently using physical, i.e. real, product models to support designers, engineers and management in their work and decision-making processes.

\subsection{Physical models and prototypes}

Generally, both the product and the manufacturing and assembly processes are today validated on the basis of physical prototypes. This is a very demanding and complex approach in terms of time requirement, funding and material expenditures (AIT 1996). In the case of microsystem technology, for instance, feasibility studies on micro assembly operations frequently require complex and lengthy test series in order to obtain the necessary process stability.

\subsection{Number of models and prototypes}

In the past, it has generally not been possible to reduce the number of models and prototypes. While rapid prototyping helped speed up the development process, there has been no decisive cost-cutting effect. The causes can be found even before the time rapid prototyping processes were introduced.

There are overlaps and interference between CAD geometry and components even during the design phase, especially at the interfaces between different design teams. While rapid prototyping can cut model generating time in this harmonisation process, there is only an insignificant decrease in the number of the co-ordination loops. Furthermore, there is still the problem of feeding the results of the study 
obtained from the hard mock-up back into the CAD model. The CAD model then becomes the basis for the improved physical model and consequently for the next configuration definition loop.

Process-relevant decision have to be made even before the existence of a CAD geometry which is required to generate an RP model. Design-related decisions are also still based on physical prototypes and can therefore in many cases be made only when it is too late.

\subsection{Employment of simulation technology in Germany}

The Institute for Machine Tools and Industrial Management of the Technische Universität München, in collaboration with the chair of production automation and production systematics at the University of Erlangen, sponsored by Bayern Innovativ $\mathrm{GmbH}$, has conducted a study on the employment of simulation technologies in German companies. This study analysed simulation tools used today, potentials available through this simulation technology and obstacles to employment of such technologies. The study distinguishes between different simulation technologies, such as workflow simulation, graphical 3D simulation, multi-body and FEM simulation (Reinhart \& Feldmann 1997).

Asked about the most important factors of success in simulation projects, the most frequent benefits named were competent implementation (35\%), followed by professional project management and a well co-ordinated specification of requirements (17\% each). The correct selection of simulation software, the quality of data sources as well as an interpretation of the calculation results in a manner understandable to decision makers seemed just as important to those questioned (Figure 1).

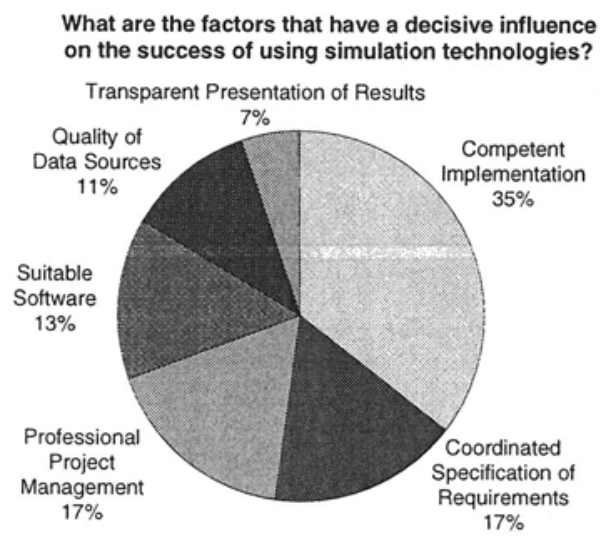

Figure 1: Success of simulation projects. 
The companies were also asked to state crucial reasons against the employment of simulation. To many, high front-end investments, the additional time expenditure during the introductory phase and a shortage of qualified staff, were deterring factors. Only few, however, doubt the benefits of simulation or do not see any suitable field of application.

Bottom line: There is little doubt among industrial companies about the benefits of simulation tools. Yet, companies shrink from employing such tools because of initial obstacles like high front-end investments and staff skill levels.

In future, however, improvements will be required not only in terms of user friendliness, user assistance in the modelling and results evaluation phase, but also with regard to the feasibility within a company through a conclusive overall strategy of digital mock-up.

\section{DIGITAL MOCK-UP - INTEGRATED MANUFACTURING SYSTEM AND PRODUCT DEVELOPMENT}

The demand in international markets is primarily driven by a higher product quality, a ,short time to market" philosophy, an increasing number of variants due to customer-oriented marketing and the growing product complexity. Considering these marginal conditions, the most important benefit of digital mock-up is the ability to drastically cut development times, enhance quality and reduce modification costs.

Owing to the increasing complexity of the products and shorter product life, it is becoming more and more necessary to fall back on existing or pre-developed concepts. Simultaneous new development of product and production will become too expensive in the future. The continuously changing global markets of the future will demand the development of new products at ever shorter intervals. But then the production facilities can no longer be economically utilised by a product owing to smaller quantities and a larger number of variants. Existing facilities and production processes must in future be re-used to an increasing extent in order to cut development time and costs. The concept of digital mock-up process simulation supports the re-use of production processes from previous projects, thus providing a quick and efficient answer to ,what if" questions of design engineers and assembly planners.

\subsection{Just-in-time with digital mock -up}

The customary hardware check (HW check, Figure 2) performed on the physical product mock-up is very time-consuming despite the use of rapid prototyping processes and may cause considerable delays during product and process development. The development activities progress as the physical product mock-up is being built (construction time, Figure 2). Later investigations performed on the hardware not only cause high costs for component or assembly modifications but also follow-up costs for unnecessary tests and modifications performed on interfacing parts or occasionally even on the entire concept. 


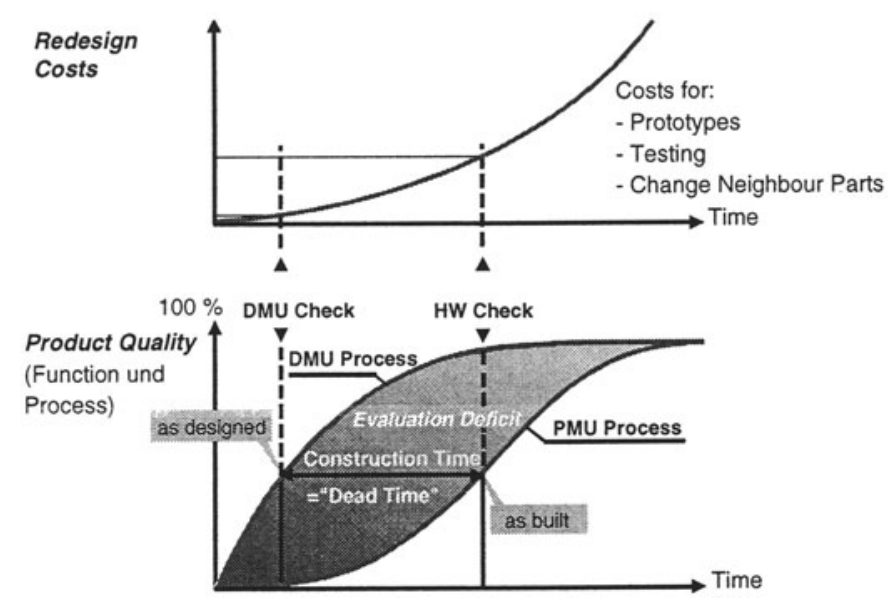

Figure 2: Just-in-time assurance.

It is only through a DMU check at the time of development (as designed, Figure 2), that a high level of development quality and a ,just-in-time“ audit of the development configuration can be ensured by avoiding expensive physical models and prototypes. Thus, a digital mock-up strategy makes for a significant reduction in development time. In today's global markets, the capability of being able to develop new products quickly and cost-effectively is the key to competitiveness in industry. Effective product development will be noticeably simplified by the potential of the DMU technology.

\subsection{Concurrent engineering}

If DMU methods and tools are adopted also by the suppliers in the automotive and aircraft industries, this will support the simultaneous engineering process between car and aircraft manufacturers and their suppliers. In future, companies using digital mock-up can work in a concurrent engineering environment, regardless of their size and product range.

The DMU system will provide genuine concurrent engineering through the coordination of parallel development activities within a team of engineers. Developers will be able to lay out, analyse and modify, taking account of product assembly requirements and production environment. Digital mock-up allows developers to go through the layout/analysis/modification process faster and with greater efficiency through rapid information loops and feedback in interdisciplinary teams. Automatic change reviews can bring about real concurrence of the work.

Change management is a key factor in a digital mock-up strategy. The versions of models and components are updated automatically. Factoring modifications to models and components into the simulation process ensures that product and process data always reflect the latest build standard. Thus, using digital mock-up, 
design engineers and production planners are able to jointly modify product and process data, thereby reducing development time.

The consistent application of simulation technology will greatly increase the data volume of companies. Today, product data mostly generated with CAD systems are managed in any EDM/PDM system. Data required to support manufacturing processes and layout of production tooling are not taken into account, though. IT systems are mostly isolated applications which do not permit any general availability of data and structured configuration management. To ensure effective and consistent employment of digital mock-ups, it is necessary to look at these marginal conditions so that these potential benefits can be fully exploited.

\subsection{Data management}

EDM systems today are widely discussed as means to meet the challenges of the future in terms of time, quality and costs in the product design, development and manufacturing process. The requirements profile of digital mock-up can be divided into the areas of document management, data maintenance, structure, configuration and workflow management. These activities include the management of data and structures of product, process and manufacturing resources.

It is especially the inter-company integration of suppliers into the product design, development and manufacturing process that places additional demands on the project management and workflow component. The project management must be able to provide information both within a project and throughout the company. Coordination among manufacturers and suppliers requires the continuous exchange of data at different development stages. The purpose of the EDM system is to relieve the user of manual, recurrent tasks, such as converting, transmitting and checkingin of data and at the same time to monitor the workflow and record it in a traceable manner.

In the EDM systems, the project-related simulation runs must be managed with references to the data and calculation standards used. Every time a modification to the product, a manufacturing process or tool is released, the relevant simulation runs must be re-verified automatically and in the batch processing mode. Once the workflow is defined, it is ensured that the right user is informed only if the calculation standards for simulation are violated.

In large projects and companies with numerous suppliers, the requirement for permanent and efficient communication between the parties involved still presents a difficult task. All findings and test results must therefore be made available to every workstation in the Intranet, using the EDM system. The Intranet, which is based on Internet technologies, is undoubtedly offering a large potential for better communication even to distributed locations. The Intranet approach ideally combines the obligation of a party to provide information in the network with the obligation of many parties to call up this information (through individual access to information in the network) (Schacher \& Tang 1996). 


\subsection{Integrated development of product and production}

The main phases in the development process are problem identification, concept definition, layout, detail design and engineering. The early phases of product development - definition of the task and proof-of-principle studies - have the greatest influence on the future result and the costs (Figure 3). The possibilities to influence the costs are far greater. However, in these early phases, cost appraisal is the most difficult task and is still at a low confidence level. This contradiction can be overstated as follows: Costs cannot be appraised reliably until it is too late for changes (Reinhart et al. 1997).

The tools used in the early phases of the product development process have been analysed. The analysis focused on the use of sketches, drawings, physical and rapid-prototyping models during product and process development. The objective was to develop the consequences to be drawn for future digital mock-up strategies on this basis. The models and prototypes above all serve the following purposes in product and process development:

- Analysis of product and process

- Appraisal of functional aspects

- Development of new concepts

Therefore, as a minimum, digital mock-up must be able to meet these requirements of designers and engineers. Moreover, the communication of analyses and evaluation results within teams, even to distributed locations, is significantly simplified. Easy-to-understand and easy-to-handle structures covering the entire product, product environment, the required tooling and the production process will substantially improve documentation and navigation through large data volumes.

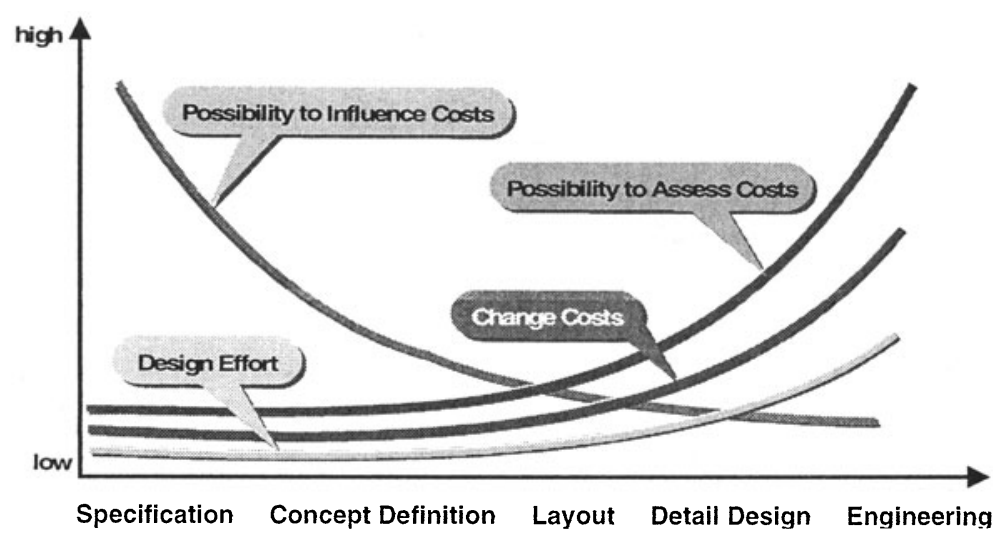

Figure 3: Development process phases

Simulation results are automatically documented during operation of the DMU system. This on-line documentation of the development standard serves as a memory aid not only for one engineer or a team but also for the whole company. 
The assembly process thus documented could for instance be re-used for simulation runs in other projects and, if necessary, be modified with relatively little effort. During the transition phase between tests and analyses performed on physical models and digital mock-ups, the simulation results must be validated against a small number of physical prototypes. The creation of prototypes will be required only at critical points in the product development process. They must be generated on the basis of specific tests and only provide substantiating data for single, specific aspects. The time pressure associated with the generation of prototypes is taken out of the development process by the preceding quick simulation on the digital mockup.

The digital mock-up becomes more detailed as the development process advances and grows from the concept all the way to the final type approval perspective. The different perspectives on different stages of product and process development serve as milestones in the development process. They define the required data quality and suitable simulation functionality of the DMU system.

The development process of the future - changed by digital mock-up strategy - can be divided into two main phases:

- First, the virtual phase, where product, manufacturing processes and production systems are developed and validated by means of computers and software.

- Second, the physical phase with the actual implementation of the product.

A digital mock-up strategy requires restructuring of the business processes to allow the efficient use of IT tools. In this connection, the interaction between business process and IT utilisation, which is not free from contradiction, plays a fundamental role: On the one hand, IT is an indispensable tool for the efficient implementation of a business process and, on the other hand, information systems, as has been increasingly the case in large companies over the past few decades, also tend to cement established workflows that no longer meet the requirements of the time (Schacher \& Tang 1996). A strategically and technically balanced and continuous evolution of the business processes and IT utilisation is increasingly recognised as one of the key factors of success in today's global competitive environment, for companies able to quickly and flexibly implement a digital mock-up strategy based on best-in-class software will gain a decisive edge on their competition.

\section{SUMMARY}

The results of the AIT initiative have shown that there is a great demand for simulation technologies especially in the automotive and aircraft industries. The just-in-time assurance of geometrical boundary conditions, tolerances, functions, manufacturing and assembly processes reduces the risk of cost-intensive, late design changes, increases the starting quality of products and decreases start-up problems in production. The employment of simulation technologies to reduce the number of prototypes - digital mock-up - is therefore one of the key technologies permitting time and cost savings that go beyond the potential of traditional 
prototyping processes. Digital mock-up permits three fundamental changes in future development processes:

- It will be possible to decisively speed up the development process. This permits a rapid review of a large variety of production scenarios, thereby increasing the flexibility of production.

- DMU supports more reliable production cost and time estimates as well as quality and risk assessment, as the simulation models are verified by real processes. This will have a strong effect on the decision-making process.

- Modelling and simulation using DMU methods and tools decisively improves flexibility in the development process.

Based on an increasing level of acceptance by the management, which results from the aforementioned benefits, digital mock-up will establish itself as a reference and communication platform for the entire product life cycle. However, the success of the digital mock-up strategy is not guaranteed only by new user-oriented IT technologies. The new potential can be fully exploited only through adjustment, but especially through organizational changes and changes in the development process.

\section{REFERENCES}

AIT 1996: Advanced Information Technology in Design and Manufacture AIT (ESPRIT project 7704) 1996.

DMU-PS 1996: Digital Mock-up Process Simulation for Product Conception and Downstream Processes DMU-PS (Brite/EuRamproject BE 95-1537) 1996.

Praun von, St.: Digital Mock-up Process Simulation. Prototyping Technology International'97, UK \& International Press, page 97-100.

Praun von, St.: Digital Mock-up - Meeting the Challenges of the Future. Prototyping Technology International October'97 Issue 2, UK \& International Press, page 72-74.

Reinhart, G.; Bichlmaier, C.; Glander, M; Praun von, St.; Reicheneder, J; Schmalzl, B.; Zanker, W.: Effiziente Produktentwicklung - Integration von Konstruktion und Montageplanung. VDI-Z 139 (1997) 4, page 40-43.

Reinhart, G.; Feldmann, K.: Simulation - Schlüsseltechnologie der Zukunft? Stand und Perspektiven. Herbert Utz Verlag, München, 1997.

Schacher, D.; Tang, T.: Einfuß neuer Informationstechnologien auf den globalen Produktentstehungsprozeß. VDI Berichte Nr. 1283, 1996.

\section{BIOGRAPHY}

Dipl.-Ing. Stefan von Praun, born 1969, received a degree in mechanical engineering at the University of Technology Munich, Germany. Since 1995 he works as research assistant at the Institute for Machine Tools and Industrial Management of Prof. Dr.-Ing. G. Reinhart and Prof. Dr.-Ing. J. Milberg. His research interests address the fields of Digital Mock-up, Simultaneous Engineering and Tolerance Simulation. 